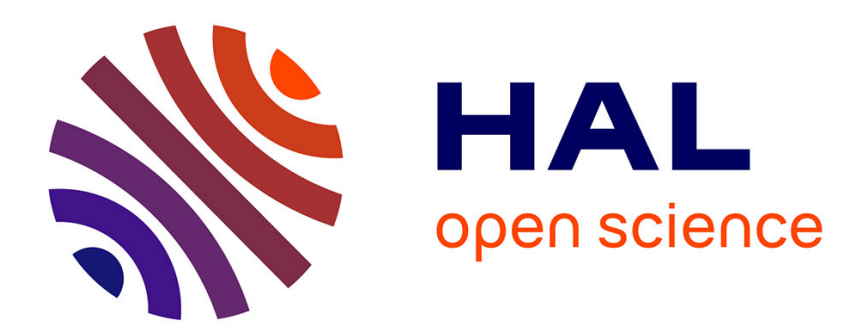

\title{
Le nonce en France au XVIe siècle, agent de diffusion de la Réforme catholique?
}

Alain Tallon

\section{To cite this version:}

Alain Tallon. Le nonce en France au XVIe siècle, agent de diffusion de la Réforme catholique?. La Réforme catholique: formes de diffusion, May 2001, Haïfa, Israël. p. 122-137. hal-02091730

\section{HAL Id: hal-02091730 \\ https://hal.sorbonne-universite.fr/hal-02091730}

Submitted on 6 Apr 2019

HAL is a multi-disciplinary open access archive for the deposit and dissemination of scientific research documents, whether they are published or not. The documents may come from teaching and research institutions in France or abroad, or from public or private research centers.
L'archive ouverte pluridisciplinaire HAL, est destinée au dépôt et à la diffusion de documents scientifiques de niveau recherche, publiés ou non, émanant des établissements d'enseignement et de recherche français ou étrangers, des laboratoires publics ou privés. 


\section{LE NONCE EN FRANCE AU XVIE SIECLE, AGENT DE DIFFUSION DE LA REFORME \\ CATHOLIQUE?}

La présente communication veut revenir sur ce qui est souvent présenté comme une évidence, à savoir le rôle majeur du représentant ordinaire du pape, le nonce, dans la diffusion de la Réforme catholique en France. On peut citer les meilleurs spécialistes, tels Bernard Barbiche : "A partir du concile de Trente, le pape s'attache à faire des nonces les agents de la Réforme catholique, tâche particulièrement délicate dans un pays comme la France où l'affrontement entre catholicisme et réforme protestante est fort rude", ou Olivier Poncet : "A l'issue du concile de Trente (...), les nonces se muent de simples diplomates qu'ils étaient avant le milieu du XVIe siècle en véritables porteurs des idéaux tridentins" 2 . Mais si tous les historiens sont d'accord sur ce rôle du nonce, il n'existe pas à ma connaissance d'étude précise qui permette de répondre à quelques questions importantes :

- Quand et comment le nonce en France se mue-t-il en agent de diffusion de la Réforme catholique ? Parler de l'époque du concile de Trente est trop vague, étant donné la durée homérique de l'événement que Paolo Sarpi soulignait avec ironie en parlant de "l'Iliade de notre temps". Non seulement le concile lui-même fut interminable, mais il faut en plus tenir compte de sa préparation et de ses conséquences immédiates. Les documents préparatoires au concile des années 1530 font en effet partie presque intégrante de cette époque, ainsi que les interprétations post-conciliaires, romaines ou borroméennes. Or, ces interprétations ne se fixent et ne se diffusent que dans les années 1570, voire 1580. "L'époque du concile" couvre donc une grande partie du XVIe siècle et ne peut en rien signifier une rupture chronologique nette.

- Quelle Réforme catholique les nonces cherchent-ils à promouvoir ? Nous n'en sommes plus en effet à une vision historiographique qui conçoit la Réforme catholique comme un mouvement unitaire, avec un programme d'action immuable et des objectifs constants. Les décrets tridentins eux-mêmes sont interprétés de façon variée, parfois même contradictoire, suivant la période et les acteurs. Si bien entendu c'est la vision romaine que défendent les représentants des papes, cette Réforme catholique romaine

\footnotetext{
${ }^{1}$ Bernard Barbiche, "La nonciature de France aux XVIe et XVIIe siècles : les nonces, leur entourage et leur cadre de vie", Kurie und Politik. Stand und Perspektiven der Nuntiaturberichtsforschung, éd. Alexander Koller, ("Bibliothek des Deutschen Historischen Instituts in Rom", 87), Tübingen, Niemeyer, 1998, p. 68.

2 Olivier Poncet, "L'édition de la correspondance des nonces en France. Histoire et avenir d'une entreprise éditoriale française”, Revue d'histoire de l'Église de France, 86, 2000, p. 636.
} 
est "plurielle" : les différents groupes qui composent la curie n'en ont pas la même conception et ces conceptions diverses ont elles-mêmes évolué au cours du siècle.

- Enfin quel a été le succès des initiatives des nonces successifs ? Ont-ils pesé sur les destinées du catholicisme français et dans quelle proportion ? L'enjeu de la réponse à cette question est de taille : la Réforme catholique telle qu'elle s'initie dans le royaume au XVIe siècle est-elle majoritairement un élément d'importation, balayant les premières initiatives locales, qui, quand elles divergent de la nouvelle norme, sont reléguées au statut de "catholicisme critique" ? Ou loin d'être des maîtres d'œuvres, les agents romains, dont le nonce n'est que le plus évident, ont-ils été des accompagnateurs ? Toute évaluation de l'influence des nonces sur le déroulement de la Réforme catholique en France est bien entendu fort difficile à réaliser, mais cette difficulté même est révélatrice de notre incapacité à penser la Réforme catholique en fonction de paramètres clairs et précis, qui existent pour les Réformes protestantes, ne serait-ce que de façon négative par la rupture du lien avec l'ancienne Église, sa hiérarchie, ses dogmes, sa liturgie. Quels paramètres peuvent faire parler de Réforme catholique ? La réception des décrets conciliaires ? Leur application ? Les concile provinciaux, les synodes diocésains, les visites pastorales ? La résidence ? L'arrivée de jésuites, de capucins, d'ursulines, de confréries du Saint-Sacrement, des Quarante-Heures ? Les séminaires ? Ou encore des éléments éminemment subjectifs comme la bonne moralité ou la bonne formation des clercs ? On pourrait multiplier les critères possibles et cette multiplicité fait de la Réforme catholique une sorte "d'auberge espagnole" historiographique - qualificatif sans allusion aux reproches des parlementaires gallicans. Cela ne doit pas forcément nous faire douter de son existence, mais peut-être de son essence.

Pour répondre aux questions posées en introduction, la source essentielle est bien entendu la correspondance des nonces en France, très largement éditée pour le XVIe siècle et donc facilement accessible, mais pour autant assez rarement utilisée par les historiens français, comme le remarquait récemment Olivier Poncet ${ }^{3}$. Parmi tous les représentants du Saint-Siège auprès des États catholiques, le nonce ordinaire envoyé au roi très chrétien avait la particularité de ne pas posséder les pouvoirs de légat a latere et donc de ne pouvoir exercer de juridiction dans le royaume, au contraire par exemple de son homologue en Espagne ${ }^{4}$. Cela ne l'empêche pas d'être plus qu'un simple ambassadeur d'un prince envoyé à un autre prince, mais réduit considérablement ses pouvoirs dans l'exercice de son autre fonction, celle de délégué apostolique. Certes, des légats a latere envoyés en France ont pu suppléer cette carence des pouvoirs du nonce,

\footnotetext{
3 Olivier Poncet, op. cit., p. 637-638.

4 Pierre Blet, "La double mission du nonce auprès du roi très chrétien", L'invention de la diplomatie. Moyen Age - Temps modernes. éd. Lucien Bély, Paris, Puf, 1998, p. 249.
} 
mais ils sont surtout envoyés pour des missions extraordinaires à caractère politique dans la première moitié du siècle et leur activité de juridiction est étroitement contrôlée par le pouvoir temporel ${ }^{5}$. Après la clôture du concile de Trente, les papes n'envoient plus guère de légats en France. Les quelques facultés spirituelles concédées au nonce en matière d'absolution ne suffisent pas à compenser l'absence de juridiction ecclésiastique, et donc de pouvoir sur l'Église gallicane ${ }^{6}$.

À défaut de pouvoirs, le nonce dispose bien sûr d'une grande influence. Ayant le pas sur tous les ambassadeurs, il est le plus souvent traité avec de grands égards par le roi, écouté avec respect sinon toujours entendu. Nombreux sont ceux qui lui font parvenir leurs doléances et souvent attendent son soutien pour des réformes ecclésiastiques. Le nonce Castelli rapporte ainsi la démarche d'un bourgeois de Paris en 1581 venu lui porter un mémoire, présenté comme venant du "popolo minuto" de Paris, sur la réforme du clergé. "Et s'il y a quelques impertinences, toutefois il y a beaucoup de bonnes choses et on découvre bien de celles là qu'il existe une grande avarice et simonie sur quelques points parmi ces curés et aussi parmi les évêques en ce qui concerne le sacrement de confirmation, ce qui est une chose importante" 7 . Ce témoignage est cependant assez exceptionnel, mais il prouve que le représentant du pape pouvait apparaître comme un recours à un peuple catholique parisien aussi anticlérical qu'intransigeant. Cependant, par sa mission même, le nonce évolue avant tout dans le monde de la cour et il doit en adopter les usages. Sa fonction d'ambassadeur l'attache à la personne du roi, ce qui limite évidemment ses possibilités de contact avec le clergé français et les fidèles.

Bien sûr, ce personnage ecclésiastique peut être mal à l'aise dans une cour laïque. "Qui si negocia a cavallo" soupire le nonce Rodolfo Pio di Carpi après une audience écourtée par le départ de François Ier à la chasse ${ }^{8}$. Agent diplomatique, le nonce peut en outre subir les conséquences des variations et sautes d'humeur dans les relations au jour le jour entre la France et Rome. Ainsi, la fin du pontificat de Grégoire XIII est marquée par le mécontentement français devant une politique pontificale jugée trop favorable à

5 Bernard Barbiche et Ségolène de Dainville-Barbiche, "Les légats a latere en France et leurs facultés aux XVIe et XVIIe siècles", Archivum Historiae Pontificae, 23, 1985, p. 93-165. Les auteurs signalent que deux nonces, en 1522 et en 1538 , reçurent les facultés de légats a latere, mais plus un seul après 1540 , Ibid., p. 98. Leurs facultés étaient de toute façon plus limitées que celles habituellement conférées aux légats, Ibid., p. 117. Le nonce Ferrerio, le dernier à recevoir ces facultés, se vit purement et simplement interdire de s'en servir par le pape lui-même, afin de ne pas nuire aux intérêts de l'administration curiale, Ibid., p. 145.

${ }^{6}$ Bernard Barbiche et Ségolène de Dainville-Barbiche, "Les pouvoirs des légats a latere et des nonces en France aux XVIe et XVIIe siècles" dans M. Maccarrone et A. Vauchez éds., Echanges religieux entre la France et l'Italie du Moyen Âge à l'époque moderne, Genève, Slatkine, 1987, p. 259277.

7 Robert Toupin éd., Correspondance du nonce en France Giovanni Battista Castelli (15811583), (“Acta Nuntiaturae Gallicae” 7), Rome-Paris, Presses de l’Université Grégorienne-E. de Boccard, 1967, p. 183.

8 Jean Lestocquoy éd., Correspondance des nonces en France Carpi et Ferrerio 1535-1540, (Acta Nuntiaturae Gallicae" 1), Rome-Paris, Presses de l'Université Grégorienne-E. de Boccard, 1961, p. 113. 
l'Espagne. Le nonce Anselmo Dandino aggrave ce mécontentement en diffusant sans autorisation royale la bulle In Coena Domini, véritable provocation pour les parlements. Le nonce subit la disgrâce royale et tous se détournent de lui. En 1581 son successeur Castelli est outré par l'accueil qui lui est fait, par comparaison avec ce qu'il avait pu observer dans les cours espagnole ou impériale, et plus particulièrement par l'attitude des clercs : aucun ne lui a fait de visite de courtoisie après son arrivée à Blois et maintenant que la cour est à Paris, l'évêque de Paris ne lui a même pas envoyé un mot de bienvenue. Castelli craint qu'une telle indifférence ne nuise au bon déroulement de sa nonciature ${ }^{9}$. Avec un mépris assez caractéristique du sentiment de supériorité éprouvé en Italie après le concile de Trente face à la "corruption" gallicane, il écrit au cardinal de Côme, secrétaire de Grégoire XIII : "Votre Seigneurie Illustrissime peut comprendre dans quelle estime se trouvent les nonces de Notre Seigneur [le pape] dans cette cour et quel fruit on peut espérer avoir avec ces prélats, qui ont tant besoin d'aide pour eux-mêmes, et pour les si grands abus que non seulement ils permettent, mais qu'ils entretiennent dans leur diocèse, s'ils ne viennent jamais visiter le nonce"10. Tous les nonces n'ont pas le même sentiment d'isolement que Castelli au début fort difficile de sa mission, mais il est certain que leur statut d'agent diplomatique, leur séjour à la cour ne leur permettent pas toujours d'intervenir avec efficacité dans la vie quotidienne de l'Église gallicane ${ }^{11}$.

L'ont-ils seulement tous voulu ? Les fragments que nous avons conservé des correspondances des premiers nonces permanents en France nous montrent des nonces avant tout soucieux de politique internationale. Dans le contexte des années 1520 où se jouent le destin de l'Italie et la survie même de l'État pontifical, quoi de moins étonnant que de voir le représentant du pape s'occuper avant tout de l'urgence politique et se soucier assez peu des cercles évangélistes, des mesures de réforme des conciles provinciaux ou même des premiers témoignages de dissidence religieuse. En l'absence de conservation de la correspondance, il est difficile d'apprécier l'action du dernier nonce en France de Clément VII, Cesare Trivulzio, après la paix de Cambrai. Nous ne voyons vraiment le nonce à l'action qu'à partir du pontificat de Paul III. Contrairement à ce qui est trop souvent avancé, la mission du nonce a déjà son double caractère politique et religieux, bien avant la conclusion du concile de Trente. Pape soucieux de réformer les abus ecclésiastiques, de réprimer l'hérésie, d'assurer la paix entre les

\footnotetext{
${ }^{9}$ Robert Toupin éd., Correspondance du nonce en France Giovanni Battista Castelli..., p. 132, Castelli au cardinal de Côme, Paris, 15 juin 1581.

${ }^{10}$ Ibid., p. 120.

11 On en trouve un bon exemple dans la correspondance de Girolamo Ragazzoni à propos des assemblées du clergé, Pierre Blet éd., Girolamo Ragazzoni évêque de Bergame, nonce en France. Correspondance de sa nonciature 1583-1586, (“Acta Nuntiaturae Gallicae” 2), Rome-Paris, Presses de l’Université Grégorienne-E. de Boccard, 1962, p. 545.
} 
princes chrétiens pour résister au Turc, Paul III modifie considérablement la politique pontificale par rapport à ses prédécesseurs Médicis. Cela n'est évidemment pas sans conséquence pour ses agents diplomatiques auprès du roi très chrétien qui doivent faire comprendre ce changement à leurs interlocuteurs. Nonce de 1535 à 1537, Rodolfo Pio di Carpi note au début de sa nonciature que les autres ambassadeurs le regardent avec curiosité car ils commencent à comprendre ce qu'est le nouveau pontificat ; mais il n'est pas encore assez clair pour eux qu'on ne négociera plus comme avant et que l'on est entré dans une nouvelle époque ${ }^{12}$.

Pour comprendre ce qu'est cette nouvelle époque, et évaluer le rôle du nonce dans la diffusion d'un idéal de Réforme catholique, j'ai relevé les thèmes abordés au cours des audiences royales pour deux nonciatures sous Paul III, celle de Rodolfo Pio di Carpi et la seconde nonciature de Girolamo Dandino, en 1546-1547, à la fin du pontificat. Au cours des 40 audiences accordées par François Ier à Pio di Carpi, 135 sujets sont ainsi évoqués que j'ai classé, un peu arbitrairement en trois rubriques : affaires politiques (78 sujets, $58 \%$ ), affaires ecclésiastiques (20 occurrences, $15 \%$ ), affaires religieuses (37 sujets, $27 \%$ ). Plus de dix ans plus tard, Dandino, au cours des vingt entretiens avec François Ier puis son successeur Henri II, aborde 90 sujets dont 49 fois des affaires politiques (54\%), 24 des questions ecclésiastiques $(27 \%)$ et 17 des problèmes religieux (19\%). La prédominance du politique est donc réelle, mais pas écrasante. Il est vrai que j'ai un peu "triché" en incluant par exemple dans le religieux le concile, qui est aussi largement un problème politique et ecclésiastique, ou le schisme anglais qui a un aspect purement temporel.

Cependant, malgré ces réserves, il faut bien constater contre l'historiographie traditionnelle l'importance relative des négociations proprement religieuses et s'interroger sur leur contenu. L'aspect proprement ecclésiastique, que j'ai voulu distinguer, concerne surtout la vie de la curie (promotions cardinalices), la juridiction pontificale, notamment en matière de dispense matrimoniale, essentielle pour les unions princières, et enfin les décimes levées sur le clergé français et leur autorisation par Rome. Les questions bénéficiales ne sont abordées que pour des faveurs personnelles (dispenses diverses, exemptions totales ou partielles des taxes curiales, etc.). Les questions plus proprement religieuses tournent essentiellement autour du concile. Le nonce et le roi se préoccupent aussi de la dissidence religieuse, mais presqu'exclusivement hors des frontières du royaume : le schisme anglais, mais aussi les tentatives de médiation de François Ier invitant Mélanchthon à Paris intéressent plus le nonce que la répression intérieure, ou tout au moins ne cherche-t-il pas trop à s'immiscer dans des affaires intérieures françaises.

12 Jean Lestocquoy éd., Correspondance des nonces en France Carpi et Ferrerio..., p. 14. 
C'est bien le sentiment qui domine pour ces nonces de la première moitié du siècle, qui ne sont pas plus "mondains" que leurs successeurs d'après 1563, mais qui respectent une sorte de devoir de réserve dans les affaires ecclésiastiques et religieuses intérieures au royaume. Ils sont capables d'évaluer leur marge de manœuvre, fort limitée face à un pouvoir royal fort et jaloux de son pouvoir. Toute mesure de réforme qui lui apparaîtrait comme imposée par une autorité extérieure, que ce soit la papauté ou le concile, n'a que peu de chance d'aboutir. On peut prendre l'exemple du décret pris par Paul III le 18 février 1547 contraignant les cardinaux à n'avoir plus qu'un seul évêché et à résigner tous les autres. Le nonce Dandino peut faire part du très mauvais accueil que cette mesure rencontre à la cour ${ }^{13}$. La mort de François Ier et les débuts du règne de Henri II repoussent la négociation pour faire accepter le décret. Le cardinal Du Bellay est un de ceux qui s'y opposent avec le plus de véhémence. En 1549, un représentant français à Rome n'hésite pas à évoquer - déjà ! - le péril communiste : si ce décret, qui enlève à leurs légitimes possesseurs des biens accordés par la faveur royale, était suivi d'effet, "il vaudroit quasi autant introduire la communauté de toutes choses ; car les Princes et tous ceux qui ont pouvoir de faire des Loix, pouroient par ce moyen abolir et annuller tous les contracts et traitez faits par leurs Prédécesseurs, et se seroit tous les jours un monde nouveau" 14 . Les nonces successifs ne s'engagent pas dans un affrontement direct stérile et laissent au pape le soin de rappeler sa volonté aux ambassadeurs et aux cardinaux français. Pour être discrète, l'action des nonces n'en est pas moins efficace : les quelques années qui suivent le décret de Paul III voient les cardinaux français résigner massivement leurs innombrables évêchés. Le nonce s'efforce d'empêcher que de nouveaux abus ne naissent de ces résignations. Le cardinal Jean de Lorraine, champion incontesté du cumul, avait reçu de François Ier en 1538 l'évêché d'Agen en vue de le donner à Hector Fregoso quand il aurait l'âge. En juin 1548, le cardinal veut le résigner à cause des décisions "du concile", en fait en raison du décret du pape. Le connétable de Montmorency demande au nonce Della Torre que Fregoso puisse retenir en personne l'évêché bien qu'il n'ait que vingt ans. Le nonce oppose les décisions du concile sur l'âge requis pour être évêque ${ }^{15}$. Finalement, Jean de Lorraine conserve Agen jusqu'à sa mort deux ans plus tard.

Si le nonce en France sous François Ier et Henri II tente bien de diffuser des mesures de réforme prises à Rome ou à Trente, il le fait avec une évidente discrétion qui contraste avec la manière beaucoup plus directe de ses successeurs dans la seconde

13 Jean Lestocquoy éd., Correspondance des nonces en France Dandino, Della Torre et Trivultio (1546-1551), ("Acta Nuntiaturae Gallicae” 6), Rome-Paris, Presses de 1'Université Grégorienne-E. de Boccard, 1966, p. 174.

14 Guillaume Ribier éd. Lettres et memoires d'Estat des Roys, Princes, Ambassadeurs et autres Ministres sous les Regnes de François premier, Henry II et François II..., Paris, François Clouzier et vesve Aubouyn, 1666, t. 2, p. 214.

15 Jean Lestocquoy éd., Correspondance des nonces en France Dandino, Della Torre et Trivultio (1546-1551)..., p. 317, Della Torre au cardinal Farnèse, Vassy, 12 juin 1548. 
moitié du siècle. Ce changement d'attitude n'est pas lié à une directive romaine : Paul III ou Jules III prennent un certain nombre de mesures de réforme et encouragent les églises nationales à adopter celles déjà votées au concile ; Paul IV n'est pas précisément un modèle de retenue et de discrétion diplomatique. Pourtant, les nonces qu'il envoie en France n'adoptent pas son ton péremptoire pour exiger l'application des décisions romaines. Ce n'est qu'à partir de 1560 que le changement de ton devient évident. Sebastiano Gualterio, nonce envoyé par Pie IV après la conjuration d'Amboise, est le premier à adopter cette attitude sermonneuse qui est celle de tous ses successeurs, ne perdant jamais une occasion de remontrer à leurs interlocuteurs français tous les abus de l'Église gallicane et de leur proposer le modèle tridentin, ou plutôt son interprétation romaine, comme unique et souverain remède. Ce changement ne tient pas non plus à la personnalité des nonces. Dans les années 1560, Pie IV, puis Pie V envoient en France des nonces qui y avaient déjà exercé cette fonction sous leurs prédécesseurs Paul III ou Jules III. Si Gualterio, Santa Croce ou Della Torre ont désormais un autre langage, ce n'est pas qu'eux-mêmes aient changé ou que la papauté ait adopté une politique radicalement différente. Simplement, les représentants de Rome n'ont plus face à eux la monarchie sûre d'elle-même de la Renaissance, mais celle inquiète et faible des guerres de religion.

Profitant de cette faiblesse nouvelle du pouvoir royal, les nonces se font les agents actifs, voire agressifs, d'une Réforme catholique romaine avant même que le concile ne se soit terminé, que ses modalités d'application ne soient fixées, que la papauté ellemême ne s'en fasse le champion. Ce fait nous renvoie à l'interprétation du concile de Trente et de toute la Réforme catholique par Paolo Sarpi comme un gigantesque bras de fer entre la papauté et les puissances temporelles catholiques, ayant pour enjeu le contrôle des Églises nationales. Pour le servite vénitien, Rome a su profiter de la moindre faiblesse des pouvoirs laïcs pour prendre l'avantage et finalement triompher. Pour polémique qu'elle soit, cette interprétation a l'avantage de poser le problème de la diffusion de la Réforme catholique non pas en termes moraux mais de rapports de pouvoir et même de rapports de force. Il ne s'agit plus de décrire le triomphe de la vertu sur le vice - ou l'inverse, suivant l'opinion de l'historien -, mais de comprendre les mécanismes du pouvoir au sein d'une Église catholique menacée.

Il ne faut cependant pas pour autant adopter sans réserve le cynisme sarpéen : les autorités romaines ont bien un idéal de réforme qu'elles tentent d'imposer en faisant progresser l'absolutisme pontifical de façon concomitante et parfois consubstantielle. Elles sont même parfois capables d'oublier leur susceptibilité au profit du seul résultat. Dès août 1560, quand le parlement menace d'entamer des procédures contre les évêques qui n'obéiraient pas à l'édit royal du 26 juillet leur ordonnant de retourner dans leur diocèse pour y résider, le nonce Gualterio s'indigne devant une telle ingérence des 
autorités laïques dans les affaires ecclésiastiques ${ }^{16}$. Mais le cardinal Borromée se montre bien plus pragmatique et s'étonne de voir le nonce s'opposer à un dessein aussi louable : peu importe quelle autorité contraindra les évêques à résider pourvu qu'ils le fassent $!^{17}$ Ce contraste entre un centre romain accommodant et ses agents sur le terrain intransigeants mérite d'être noté, même si on ne peut en faire une loi. De plus en plus, la papauté donne raison à ses nonces quand ils s'opposent aux règlements laïcs en matière ecclésiastique, fût-ce pour la bonne cause. Le cas le plus évident est celui de la grande ordonnance de réforme de Blois, issue des doléances des États généraux de 1576, mais rédigée seulement en 1579. L'ordonnance reprend de nombreuses dispositions du concile de Trente, mais cette fois le pouvoir pontifical et ses représentants ne sont plus disposés à laisser le roi imposer de sa propre autorité des mesures inspirées par la législation conciliaire. Les nonces Castelli, puis Ragazzoni se conforment scrupuleusement à leurs instructions et demandent à plusieurs reprises le retrait de l'ordonnance ${ }^{18}$. Ils surveillent aussi avec inquiétude toute nouvelle velléité de réforme, comme celle que laisse présager l'assemblée de Saint-Germain réunie par Henri III à la fin de l'année 1583. Analysant les débats de Saint-Germain, l'éditeur de la correspondance de Ragazzoni, le père Pierre Blet, traduit bien le paradoxe de la position du nonce : "Salutaires en soi, peut-être, ces réformes risquaient de se trouver viciées dans leur principe, issues qu'elles seraient du pouvoir laïque"19. La lutte pour la résidence, contre la confidence, la simonie, objectifs des plus louables, ne doivent pas être des prétextes pour maintenir ou développer la tutelle du pouvoir temporel sur l’Église gallicane.

La diffusion de la Réforme catholique apparaît donc dans la correspondance du nonce comme un problème largement politique, même si les représentants du pape tentent le plus souvent de le placer uniquement sur le terrain religieux. L'interminable question de la réception des décrets du concile de Trente est à ce sujet un bon exemple. Sans entrer dans le détail des négociations, déjà bien connu ${ }^{20}$, il faut cependant insister sur un fait : les nonces ne veulent pas tant la réception des décrets que leur réception inconditionnelle, sans aucune clause de réserve concernant les libertés gallicanes ou les privilèges du roi. Elle seule marquera à leur yeux l'indépendance de la sphère ecclésiastique et surtout la supériorité du pouvoir romain sur tout pouvoir local, que ce soit celui de l'Église gallicane ou du roi. La question de l'application devient alors

16 Jean Lestocquoy éd., Correspondance des nonces en France Lenzi et Gualterio, légation du cardinal Trivultio (1557-1561), (“Acta Nuntiaturae Gallicae” 14), Rome-Paris, Université Pontificale Grégorienne, Ecole Française de Rome, 1977, p. 249.

17 Ibid., p. 255.

sq.

18 Robert Toupin éd., Correspondance du nonce en France Giovanni Battista Castelli..., p. 64

19 Pierre Blet éd., Girolamo Ragazzoni..., p. 37.

${ }^{20}$ Le livre essentiel reste celui de Victor Martin, Le gallicanisme et la Réforme catholique. Essai historique sur l'introduction en France des décrets du concile de Trente (1563-1615), Genève, SlatkineMegariotis Reprints, 1975 (réimpression de l'édition de Paris, 1919). 
secondaire. Cette dissociation entre combat pour la réception et pour l'application est essentielle pour comprendre l'action du nonce comme agent de la Réforme catholique. Il se soucie finalement assez peu de relever les tentatives du clergé français et de la monarchie pour adopter des éléments du dispositif tridentin, tentatives plus précoces et moins vaines qu'on ne l'a trop souvent dit en adoptant justement le point de vue des nonces. Ces tentatives aboutissent, aux yeux des Français, à des résultats qui valent ceux des "modèles" méditerranéens et sont la preuve de la vanité du débat sur la réception du concile. En 1579, Anselmo Dandino signale que pour le roi, le Parlement, la Sorbonne, la réforme de l'Église gallicane peut se faire sans la réception officielle des décrets, car les Français ont des cardinaux aussi bons que Borromée ou Paleotti ${ }^{21}$. $\mathrm{Si}$ on signale les qualités du clergé français, on souligne aussi volontiers les travers qui subsistent dans des pays ayant reçu officiellement le concile. Dans un entretien avec le nonce Ragazzoni, Claude d'Angennes, évêque de Noyon, “pour défense des nombreux désordres qui se produisent ici en matière de bénéfices et en ce qui concerne la discipline ecclésiastique, dit que les choses ne se passaient guère mieux en Italie, où le concile est publié" 22 . Les nonces convaincus de la corruption gallicane peuvent difficilement entendre un tel message, mais il est plus surprenant de voir comment toute l'historiographie a repris jusqu'à une période récente le point de vue romain qui lie étroitement réception et application du concile ${ }^{23}$. Or, la première question relève exclusivement d'un débat juridictionnel entre affirmation de la suprématie pontificale ou conservation des privilèges royaux ou bien d'un conflit politique entre clans catholiques intransigeant et modéré au sein même de la cour. Les nonces successifs pèsent de tout leur poids dans ce débat, mais il n'a qu'un rapport lointain avec la diffusion réelle de la Réforme catholique.

Dans ce domaine, les interventions du représentant pontifical sont nettement moins fracassantes. On peut le constater sur deux exemples précis : les conciles provinciaux et les nominations épiscopales. Le concile de Trente ordonne la réunion régulière de conciles provinciaux, disposition qui n'est appliquée que très rarement dans l'Europe catholique, à l'exception notable de la province de Milan sous le gouvernement de Charles Borromée. Ailleurs, le plus souvent, un concile unique se réunit pour adopter les mesures tridentines et leur ajouter des dispositions concernant leurs modalités d'application. En France, juste après la clôture du concile de Trente, seul le cardinal de Lorraine réunit un concile à Reims, dans la plus grande indifférence du nonce Santa

${ }^{21}$ Ivan Cloulas éd., Correspondance du nonce en France Anselmo Dandino (1578-1581), (“Acte Nuntiaturae Gallicae” 8), Rome-Paris, Presses de l'Université Grégorienne-Editions de Boccard, 1970, , p. 452-453.

22 Pierre Blet éd., Girolamo Ragazzoni..., p. 482, lettre au cardinal Rusticucci Paris, 25 novembre 1585 .

23 Voir le constat dressé par Marc Venard, Le catholicisme à l'épreuve dans la France du XVIe siècle, Paris, Éditions du Cerf, 2000, notamment p. 286. 
Croce. Son exemple n'est repris dans les autres provinces de l'Église gallicane qu'au début des années 1580, sans que les nonces successifs n'aient vraiment cherché à encourager les archevêques français à remplir les obligations tridentines en ce domaine. Ce manque de zèle des agents romains en faveur des conciles provinciaux n'est d'ailleurs pas particulier à la France. Quand le cardinal de Bourbon réunit enfin le concile de sa province de Rouen en 1581, l'enthousiasme du nonce Castelli est plus que modéré. Les décrets normands "n’ont pas la majesté ni le sérieux ("gravità") des conciles milanais"; beaucoup de dispositions ne semblent pas relever de la compétence des conciles provinciaux ${ }^{24}$. La seule chose qu'apprécie le nonce est de voir le cardinal de Bourbon soumettre les décrets à l'approbation du pape, même s'il déplore de le voir faire la même chose avec le roi. Une fois de plus, le conflit de juridiction semble occulter complètement les dispositions elles-mêmes. Il est vrai que ce conflit de juridiction peut compromettre l'application des mesures décidées au concile : quand Castelli conseille à l'archevêque de Lyon Pierre d'Épinac de réunir un concile provincial, le prélat se montre sceptique sur son utilité, car le parlement risque de s'ingérer et de censurer les décrets ${ }^{25}$. D'initiative locale, soumis au contrôle du pouvoir temporel, les conciles provinciaux n'ont rien pour séduire les représentants de Rome. On peut multiplier les exemples de leur désintérêt, y compris chez les nonces les plus actifs en faveur de la Réforme catholique. Girolamo Ragazzoni par exemple transmet sans commentaire une demande de Monsieur en faveur du chapitre collégial de SaintMartin de Tours : les chanoines craignent une limitation de leur exemption par le concile provincial et implorent la protection de Rome ${ }^{26}$. L'archevêque de Bourges se plaint que ses suffragants boudent son concile provincial et demande au nonce d'intervenir auprès du roi et du pape pour les contraindre d'y assister. Ragazzoni préfère esquiver et ne pas s'engager ${ }^{27}$. La seule chose à laquelle les nonces s'intéressent vraiment est l'envoi des décrets à Rome par les métropolitains pour obtenir confirmation et éventuellement correction.

En ce qui concerne les nominations épiscopales, on observe un réel changement après 1560 et on voit les nonces morigéner plus volontiers les rois et leur rappeler leurs devoirs. Mais il leur est difficile d'aller plus loin, car ils n'ont aucun rôle institutionnel dans le processus mis en place par le concordat de 1516, avant l'établissement d'une nonciature permanente. Lors des débats à Trente, il avait bien été proposé de confier aux nonces le soin d'enquêter sur les candidats à l'épiscopat, mais cette proposition avait été rejetée avec vigueur par l'épiscopat français. Ce n'est que par la bulle de Grégoire XIV Onus apostolicae servitutis du 15 mai 1591 que Rome confie aux légats $a$

\footnotetext{
24 Robert Toupin éd., Correspondance du nonce en France Giovanni Battista Castelli, p. 150

25 Ibid., p. 183

26 Pierre Blet éd., Girolamo Ragazzoni..., p. 186-7, Paris, 27 janvier 1584.

27 Ibid., p. 263.
} 
latere et aux nonces le soin d'établir les procès de promovendis ${ }^{28}$. Mais cette mesure valable pour toute la chrétienté n'est pas reconnue dans la France gallicane.

En ce qui concerne la période étudiée ici, les interventions du nonce sont donc ponctuelles et relèvent de la diplomatie d'influence. Il peut mettre en garde le roi ou le pape sur les médiocres qualités d'un candidat à l'épiscopat et bloquer ainsi le processus de nomination. Force est de constater que ces interventions sont rares, y compris chez les nonces les plus zélés. Prenons l'exemple des nonces Giovanni Battista Castelli (1581-1583) et Girolamo Ragazzoni (1583-1586) qui représentent sans aucun doute le modèle du nonce voulant œuvrer à la diffusion de la Réforme catholique. Tous deux issus de ce groupe d'évêques borroméens de choc qui a mis en application la Réforme tridentine en Italie du Nord, ils ont pour interlocuteur un roi affaibli politiquement et de plus en plus dévot, doublement sensible donc aux pressions du représentant de Rome. Malgré cette situation favorable, malgré leur zèle, les interventions précises de Castelli et de Ragazzoni restent rares. Au delà des sermons généraux sur les devoirs du roi, dont ils ne se privent pas, on ne peut remarquer que quelques cas où ils s'opposent à une nomination royale précise : Saluces, où il s'agit non pas de défendre la Réforme catholique, mais d'empêcher le roi de nommer à un évêché qui ne relève pas des dispositions concordataires ${ }^{29}$; Verdun où certes le candidat est indigne, mais qui surtout relève exactement du même cas que Saluces ${ }^{30}$, et enfin Lisieux où Ragazzoni dénonce la candidature d'un confidentiaire ${ }^{31}$. Mais s'il le fait, alors qu'il ne s'agit certainement pas du seul cas survenu pendant sa nonciature, c'est parce que ce confidentiaire agit pour le compte d'un favori de Monsieur, que la nomination vient de ce dernier et que finalement Henri III est trop heureux d'opposer à son frère les réticences romaines. Le nonce peut donc à la fois contrecarrer la volonté d'un prince mal vu à Rome pour ses alliances avec les hérétiques et empêcher la pratique détestable de la confidence, sans pour autant déplaire au souverain.

La prudence, le choix très ciblé des interventions semblent caractériser l'action du nonce en matière de nomination épiscopale. Mais on retrouve ces même traits pour d'autres aspects essentiels de la Réforme catholique. Ragazzoni intervient vigoureusement pour envoyer l'évêque de Saluces résider dans son diocèse ${ }^{32}$ : il s'agit d'affirmer l'autorité d'un représentant du pape sur le titulaire d'un évêché italien. Castelli comme Ragazzoni font des instances auprès d'Henri III pour obtenir son accord à des visites apostoliques, mais il s'agit à nouveau seulement de visites à Saluces ou

28 Bernard Barbiche et Ségolène de Dainville-Barbiche, "Les pouvoirs des légats a latere et des nonces en France aux XVIe et XVIIe siècles”, op. cit., p. 269.

29 Robert Toupin éd., Correspondance du nonce en France Giovanni Battista Castelli..., p. 216.

30 Pierre Blet éd., Girolamo Ragazzoni..., p. 305.

31 Ivan Cloulas, "Les rapports de Jérôme Ragazzoni, évêque de Bergame avec les ecclésiastiques pendant sa nonciature en France (1583-1586)", Mélanges d'archéologie et d'histoire de l'École française de Rome, 72, 1960, p. 518.

32 Pierre Blet éd., Girolamo Ragazzoni..., p. 165, p. 206. 
dans les Trois Évêchés. Quel que soit le sujet sur lequel ils interviennent, Réforme catholique et défense de la juridiction pontificale sont indissociables dans l'action des nonces.

Cet exposé trop rapide permet néanmoins de tirer quelques conclusions qui ont valeur générale. Le nonce en France a bien été un agent de diffusion de la Réforme catholique, mais la nature de son action, son caractère finalement très limité même après 1560 sont révélateurs du fait qu'il n'est pas l'initiateur, ni même un des acteurs importants de cette Réforme catholique française. Le nonce a surtout voulu affirmer la suprématie pontificale en profitant après 1560 de l'affaiblissement de la monarchie. Certes, cette affirmation n'est justement possible que par la promotion de la Réforme catholique. Dans les faits, ce combat passe par deux types d'actions bien différents : des affirmations générales flamboyantes, mais sans grandes conséquences, et des interventions ponctuelles, calculées avec réalisme. L'ambition des secondes est d'ailleurs fort modeste. Le nonce semble ne pouvoir influencer qu'à la marge le cours de la Réforme catholique dans le royaume.

Cette constatation permet de mieux comprendre les formes de diffusion de la Réforme catholique en France. Loin d'être une importation romaine ou borroméenne, elle est avant tout un phénomène né des appétits réformistes de la fin du XVe siècle, poursuivi par l'évangélisme, puis par les premières mesures de Contre-Réforme dont témoignent les conciles provinciaux de 1528. En France comme ailleurs, le cadre de la Réforme catholique a été avant tout national et il est temps d'en finir avec l'opposition traditionnelle entre un protestantisme "national" et un catholicisme "international" imposé par Trente et Rome. Mais si l'action romaine, et en particulier celle des nonces, n'a pas eu un caractère initial et décisif, il n'en reste pas moins qu'elle a maintenu une forme de coordination entre les différentes Réformes catholiques nationales, que l'affirmation obstinée de la juridiction pontificale, dans la majesté de son principe tout comme dans la trivialité de ses prétentions, a su, en France comme ailleurs, maintenir un équilibre difficile entre l'universel et le national, entre centralité romaine et dynamisme local.

\section{Alain TALLON}

